\title{
A New Protocol for Determining Intervention with Trans-Catheter Arterial Embolization or Surgical Hemostasis for Obstetric Hemorrhage
}

\author{
Mai Myoga', Kazuaki Yoshimura2*, Toru Hachisuga ${ }^{1}$ \\ ${ }^{1}$ Department of Obstetrics and Gynecology, University of Occupational and Environmental Health, \\ Kitakyushu, Japan \\ ${ }^{2}$ Wakamatsu Hospital, University of Occupational and Environmental Health, Kitakyushu, Japan \\ Email: ${ }^{*}$ yoppy@med.uoeh-u.ac.jp
}

Received 9 January 2015; accepted 22 February 2015; published 27 February 2015

Copyright (C) 2015 by authors and Scientific Research Publishing Inc.

This work is licensed under the Creative Commons Attribution International License (CC BY). http://creativecommons.org/licenses/by/4.0/

\section{(c) (i) Open Access}

\section{Abstract}

Objectives: The aim of this study is to introduce protocols for choosing trans-arterial embolization (TAE) or surgical hemostasis as an initial therapy for obstetric hemorrhage. Materials and Methods: From 2002 to 2011 at our hospital, the medical records of the patients who underwent TAE or surgical hemostasis for obstetric hemorrhage were reviewed to assess the following data: The causes of obstetric hemorrhage, Shock Index (SI) and obstetrical disseminated intra-vascular coagulation (DIC) score, amount of bleeding, transfusion, and operation time. Results: Twenty-five patients underwent TAE and six underwent surgical hemostasis. SI and obstetrical DIC score of the TAE group were $1.0(0.4-2.2)$ and $6.0(1-32)$, respectively. They were significantly lower than those of the surgical hemostasis group (SI: 1.6 , obstetrical DIC score: $12.5, p<0.05$ ). Though the hemorrhage could be controlled sufficiently in 23 cases of the TAE group, 5 cases went into shock during TAE. The SI and obstetrical DIC score of shock group were $1.2(1-2)$ and $10(2$ - 32), respectively. Conclusion: Though TAE is a useful therapy to control obstetric inevitable hemorrhage, special attention should be paid to the vital signs during TAE, especially in cases where SI and/or obstetrical DIC score are higher than 1.2 and 10, respectively.

\section{Keywords}

Obstetric Hemorrhage, Trans-Catheter Arterial Embolization (TAE), Surgical Hemostasis, Obstetrical DIC Score, Complications

\footnotetext{
${ }^{*}$ Corresponding author.
} 


\section{Introduction}

Obstetric hemorrhage is one of the major causes of maternal morbidity and mortality [1]-[3] and its management represents a critical concern to obstetricians. In 2011, one third of the causes of maternal death was obstetric hemorrhage in Japan (http://www.jaog.or.jp/all/document/botai_2011.pdf). There are many treatments to control obstetric hemorrhage, i.e., uterine fundal massage, uterine bimanual compression, uterotonic agents, intra-uterine balloon tamponade, uterine packing, and placental tissue removal. If these conservative treatments are not effective, invasive interventions are required.

The trans-catheter embolization (TAE) of uterine arteries for obstetric hemorrhage was first reported in 1979 by Brown et al. [4] and the recent success rate of UAE exceeds 90\% [5]-[10] and goes up to 100\% [11]. TAE is an excellent method of preserving the uterus for future pregnancies. The usages of TAE are wide ranging, which includes postpartum hemorrhage (such as uterine atony), placenta accreta, puerperal hematoma, early pregnancy bleeding, placental polyp, and cervical pregnancy. During massive bleeding with unstable vital signs, the surgical hemostasis (including internal iliac artery ligation or hysterectomy with intensive care by the anesthesiologist) is advisable. However, we sometimes encountered technically difficult cases for TAE went into shock during TAE. In addition, surgical hemostasis after unsuccessful TAE results in much more blood loss due to hemostasis completion delay. At present, as an initial therapy, there is no standard protocol for choosing TAE or surgical hemostasis to control obstetric hemorrhage. It is important to select the appropriate initial therapy in each case by carefully considering the risks and benefits. The purpose of this study is to, by reviewing the past cases in our hospital, introduce protocols for choosing TAE or surgical hemostasis as an initial therapy for obstetric hemorrhage.

\section{Materials and Methods}

The cases included in this study are from between July 2002 and February 2012. They consisted of 25 cases of TAE and 6 cases of surgical hemostasis for obstetric bleeding. We focused on the causes of obstetric hemorrhage and post-embolization complications. The medical records were reviewed to assess the following data: age, parity, emergency transport (y/n), Shock Index (SI), obstetrical DIC score [12] before TAE, volume of bleeding before and during therapy, total volume of bleeding, units of blood transfusion (amount of packed red cells, fresh frozen plasma and platelets), operation time, and hospitalization period.

The shock index (SI) is calculated as the pulse rate divided by the systolic blood pressure (mmHg). It is used as an accurate indicator to estimate the blood loss volume.

"SI = 1 and 1.5" means that blood loss volume will be about 1.5 and 2.5 litters, respectively. Obstetrical disseminated intravascular coagulation score (Obstetrical DIC score) is related with the coagulation disorder for pregnant women. This is an excellent scoring system for DIC in pregnant women which gives much weight to the underlying diseases (Table 1). It can allow us the quick and convenient diagnosis to determine the timing of starting intensive care before obtaining the blood test results. The intensive care for DIC should be given when Obstetrical DIC score is over 8 points. Over 13 points of obstetrical DIC score means that the patient is already in DIC status. SI and obstetrical DIC score are established and widely used in Japan.

At our hospital, patients undergo TAE within forty five minutes after their obstetrician's decision. All TAE were performed by two trained interventional radiotherapists. Absorbable gelatin sponge pledgets and/or microcoils were used for this process. The embolus materials were injected into the uterine arteries via right transfemoral catheters. A post-embolisation selective angiogram was used to confirm uterine arteries occlusion completion.

Because two anesthesiologists and operation room staff were always available, all of the operations began within 30 minutes. The surgical procedures were determined by attending doctors in each case.

Written informed consent was obtained from all patients and their families (regarding the risks and benefits of TAE). Statistical analysis was performed by using StatView 5.0 and p value of less than 0.05 was considered to be statistically significant.

\section{Results}

The causes of obstetric hemorrhage in the TAE group and surgical hemostasis group are stated in Table 2. "Others" in the TAE group include false aneurysm and hydatidiform mole coexisting with a fetus. "Others” in 
Table 1. Obstetrical disseminated intravascular coagulation score (obstetrical DIC score).

\begin{tabular}{|c|c|c|c|c|c|}
\hline Underlying Diseases & Points & Clinical Symptoms & Points & Blood Tests & Points \\
\hline 1. abruptio placenta & & 1. actue renal dysfunction & & 1) fibrin degradation product $\geq 10 \mathrm{mg} / \mathrm{dl}$ & 1 \\
\hline 1) uterine regidity, dead fetus & 5 & 1) anuria $(\leq 5 \mathrm{ml} / \mathrm{h})$ & 3 & 2) platelet count $\leq 10 \times 10^{4} / \mathrm{mm}^{5}$ & 1 \\
\hline 2) uterine regidity, alive fetus & 4 & 2) oliguria $(<5, \leq 20 \mathrm{ml} / \mathrm{h})$ & 4 & 3) fibrinogen $\leq 150 \mathrm{mg} / \mathrm{dl}$ & 1 \\
\hline 3) diagnosed by US and/or CTG & 4 & 2. acute respiratory failure & & 4) prothrombin time $\geq 15 \mathrm{sec} .(\leq 50 \%)$ & 1 \\
\hline 2. amniotic embolism & & 1) artificial ventilation & 4 & $\begin{array}{l}\text { 5) erythroctyte esdimentation rate } \leq 4 \mathrm{~mm} / 15 \mathrm{~min} \text { or } \\
\leq 15 \mathrm{~mm} / 15 \mathrm{~min}\end{array}$ & 1 \\
\hline 1) artificial ventilation & 3 & 2) oxygen administration & 1 & 6) bleeding time $\geq 5 \mathrm{~min}$ & 1 \\
\hline 2) assisted ventilation & 2 & 3. severe organ failure & & $\begin{array}{l}\text { 7) others (AT- } 3 \leq 18 \mathrm{mg} / \mathrm{dl} \text { or } \leq 60 \% \text {, prekallikrein, } \\
\text { a2-PI, plasminogen, other coagulation factors } \leq \\
50 \% \text { ) }\end{array}$ & 1 \\
\hline 3) oxygen administration & 1 & 1) heart (rate or foamy sputum) & 4 & & \\
\hline 3. DIC puerperal hemorrhage & & 2) liver (visible jaundice) & 4 & & \\
\hline 1) incoagulable blood & 4 & 3) brain (coma or convulsion) & 4 & & \\
\hline 2) over 2L of blood loss & 3 & $\begin{array}{l}\text { 4) digestive organ (necrotizing } \\
\text { enterocolitis) }\end{array}$ & 4 & & \\
\hline 3) 1-2L of blood loss & 1 & 4. hemorrhagic tendency & & & \\
\hline 4. eclampsia & 4 & $\begin{array}{l}\text { 1) mactotic hematuria, melena, } \\
\text { purpura, ulorrhagia }\end{array}$ & 4 & & \\
\hline \multirow[t]{5}{*}{ 5. others } & 1 & 5. others & & & \\
\hline & & 1) pulse rate $\geq 100 / \mathrm{min}$ & 1 & & \\
\hline & & $\begin{array}{l}\text { 2) systolic blood pressure } \leq 90 \\
\text { mmHg }\end{array}$ & 1 & & \\
\hline & & 3) clammy sweat & 1 & & \\
\hline & & 4) paleness & 1 & & \\
\hline
\end{tabular}

Table 2. Indications of trans-arterial embolization (TAE) and surgical hemostasis for obstetric hemorrhage.

\begin{tabular}{ccc}
\hline & TAE $(\mathrm{n}=25)$ & Surgical Hemostasis $(\mathrm{n}=6)$ \\
\hline Uterine atony & 13 & 3 \\
Ectopic pregnancy & 3 & 0 \\
Placenta accreta & 2 & 0 \\
Placental polyp & 2 & 0 \\
Placenta previa & 2 & 0 \\
Abruptio placenta & 1 & 2
\end{tabular}

the surgical hemostasis group include uterine rupture and marginal sinus bleeding of placenta. The surgical procedures included 4 cases of total abdominal hysterectomy and 2 of internal iliac artery ligation. The characteristics of the TAE cases compared to those of the surgical hemostasis cases are shown in Table 3. The SI of the surgical hemostasis group was significantly higher than that of the TAE group and the obstetrical DIC score of the surgical hemostasis group tended to be higher than that of the TAE group. The general condition of the TAE group was better than that of the surgical hemostasis group and the success rate of TAE was 92\% (23/25 cases).

There were two cases of TAE failure in our study. Case 1 was a twin pregnancy. The patient was transferred to our hospital because of uterine atony during cesarean section at the primary hospital. To control atonic bleeding, gauze was packed into the uterus. The SI and the obstetrical DIC score at the arrival at our hospital was 0.9 and 9, respectively. A TAE of bilateral uterine arteries was then performed. Eventually, we also performed a la- 
parotomy and removed the packed gauze. Atonic bleeding continued during laparotomy and therefore a hysterectomy became necessary. It was presumed that the gauze packing made it difficult to detect the bleeding during TAE. The patient in Case 2 underwent a cesarean section due to eclampsia at our hospital. The SI and the obstetrical DIC score were 2 and 32, respectively. Postoperative massive bleeding continued and a TAE was performed. Bleeding was uncontrollable and the rectus muscle of the abdomen was detected as the bleeding point via laparotomy.

Intensive care was required for 5 cases (20\%) who fell into shock during TAE. The average age of the shock group was significantly higher than the non-shock group $(\mathrm{p}<0.05)$. The total volume of the bleeding of the shock group was significantly larger than that of the non-shock group $(\mathrm{p}<0.05)$. The SI and the obstetrical DIC score before the TAE in the shock group were 1.2 and 10, respectively, which tended to be higher than those of the non-shock group (SI: 0.8, obstetric DIC score: 6) (Table 4). Major complications were pain $(n=15)$, fever (over $\left.38^{\circ} \mathrm{C}\right)(n=9)$, secondary amenorrhea $(n=2)$, and uterine necrosis $(n=1)$.

The data of 15 out of 25 cases regarding fertility after TAE were obtained. Thirteen pregnancies across seven women have been confirmed to date. First trimester miscarriages occurred in 7 pregnancies. There were 6 successful births, 2 of which had fetal growth restriction.

Table 3. Characteristics of the TAE and surgical hemostasis group.

\begin{tabular}{cccc}
\hline & TAE $(\mathrm{n}=25)$ & Surgical Hemostasis $(\mathrm{n}=6)$ & $\mathrm{p}$ Value \\
\hline Age (y.o.) & $33(22-43)$ & $31(22-42)$ & NS \\
Primipara & $6(24 \%)$ & $4(66 \%)$ & NS \\
Emergency transfer & $18(72 \%)$ & $5(83 \%)$ & $<0.02$ \\
Shock index & $1.0(0.4-2.2)$ & $1.6(0.8-2.6)$ & NS \\
Obstetric DIC score & $6.0(1-32)$ & $12.5(9-17)$ & NS \\
Bleeding before therapy (ml) & $1971(290-5562)$ & $3005(400-6500)$ & $<0.01$ \\
Bleeding during operation (ml) & $490(70-9427)$ & $3790(2500-4600)$ & NS \\
Total bleeding (ml) & $2471(290-13807)$ & $7140(2900-11000)$ & NS \\
Transfusion RCC (IU) & $6(0-80)$ & $21(4-28)$ & NS \\
FFP (IU) & $8(0-111)$ & $15(3-20)$ & $10(0-20)$ \\
PLT (IU) & $0(0-100)$ & &
\end{tabular}

RCC: Red Cell Concentrates; FFP: Fresh Frozen Plasma; PLT: Platelet Concentrate.

Table 4. Characteristics of the cases with and without shock during TAE.

\begin{tabular}{|c|c|c|c|}
\hline & Shock Group $(\mathrm{n}=5)$ & Non-Shock Group $(\mathrm{n}=20)$ & $\mathrm{p}$ Value \\
\hline Age (у.о.) & $37.2(33-43)$ & $32.2(22-40)$ & $<0.05$ \\
\hline Shock index & $1.2(1-2)$ & $0.8(0-2.2)$ & NS \\
\hline Obstetric DIC score & $10(2-32)$ & $6(0-18)$ & NS \\
\hline Bleeding before TAE (ml) & $1779(682-4380)$ & $1068(100-3002)$ & NS \\
\hline Bleeding during TAE (ml) & $1660(340$ - 9427) & $305(70-2051)$ & $<0.05$ \\
\hline Total bleeding (ml) & $3973(1878-13807)$ & $1888(120-7000)$ & NS \\
\hline Operation time (min.) & $70(50-90)$ & $61(18-118)$ & NS \\
\hline Transfusion RCC (IU) & $14(6-80)$ & $8(0-22)$ & NS \\
\hline FFP (IU) & $9(0-111)$ & $3(0-30)$ & NS \\
\hline PC (IU) & $0(0-100)$ & $0(0-40)$ & NS \\
\hline
\end{tabular}

RCC: Red Cell Concentrates; FFP: Fresh Frozen Plasma; PLT: Platelet Concentrate. 


\section{Discussion}

In this study, we reviewed obstetric hemorrhage cases. The success rate of TAE was $92 \%$ which was similar to the reported results [5] [7]-[9] [11]. TAE is a crucial method for preserving future fertility. However, we experienced 5 patients went into shock during TAE with unstable hemodynamics (SI > 1.2 and/or DIC score > 10) at the beginning of TAE. These cases required more than average blood transfusions and effort to improve their hemodynamics. In cases of severe coagulation disorders and unstable vital signs, we propose selecting surgical hemostasis under the intensive care of anesthesiologists.

Sentilhes et al. reported 11 cases of TAE failure out of a total of 100 cases. They concluded that the only factors significantly associated with failed pelvic arterial embolization were a higher rate of estimated blood loss (more than $1500 \mathrm{ml}$ ) and more than 5 transfused red blood cell units [13]. Bros et al. reported that 194 cases underwent TAE for postpartum hemorrhage and 12 cases experienced recurrent bleeding within 24 hours after TAE. Multivariate analysis revealed that presence of coagulation disorders, anatomic variations of the uterine artery vasculature and primiparity were a statistically significant risk factor for recurrent bleeding within 24 hours after uterine artery embolization for severe postpartum hemorrhage [14].

Maassen et al. reported a case of TAE for atonic uterus after normal delivery. There was persistent bleeding after TAE therefore re-TAE was performed. Gelatin sponges were injected into both internal iliac arteries, however, the woman continued to bleed and an emergency hysterectomy was required. The reason of persistent bleeding was continued blood supply from the ovarian arteries to the uterus. In this case, it was important to check the accessory blood supply [15].

Regarding the complications of TAE, Soncini et al. reported that there were hematoma at the insertion site, pain, fever, and nausea as post-embolization complications [8]. It was reported that uterine necrosis and secondary amenorrhea are rare. In this study, we found pain (60\%), fever (36\%), secondary amenorrhea (8\%), and uterine necrosis (4\%). In one case of uterine necrosis a patient underwent hysterectomy 40 days after TAE (Gelsponge as the embolus material). There were a few reports about uterine necrosis after TAE for obstetric hemorrhage. Cottier [16] reported a case of ischemic uterine necrosis after TAE with polyvinyl alcohol and Gelfoam particles. In the management of post-partum bleeding by arterial embolization, interventional radiologists and gynecologists should be aware of this complication and use polyvinyl alcohol rather than Gelfoam. Regarding the fertility after TAE, Hardeman compared 53 patients exposed to embolization with 106 non-exposed patients and concluded that the embolization does not alter subsequent fertility [17]. In our study, about half of the patients after TAE gave birth. For patients' sake, better information regarding their subsequent chances of fertility and the potential risks should be provided.

The flowchart for massive obstetrical bleeding is shown in Figure 1. After an assessment of the etiology,

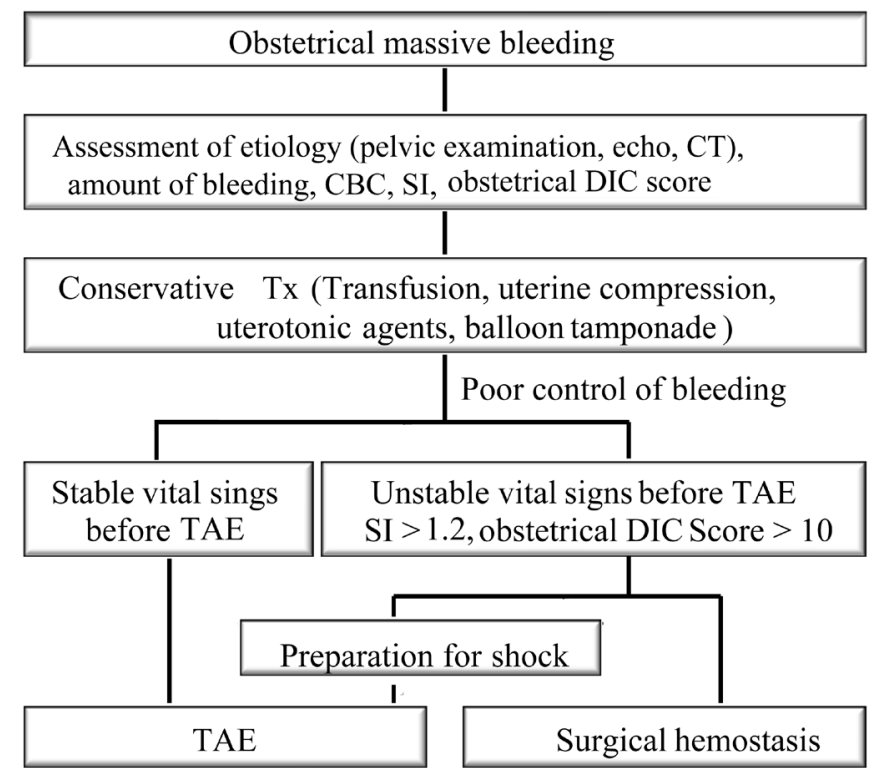

Figure 1. The proposed protocol for intervention determination. 
amount of bleeding, complete blood count, the SI, and the obstetrical DIC score for each case, a TAE should be performed for the cases with stable vital signs before the therapy. For the cases with unstable vital signs of SI > 1.2 and/or obstetrical DIC score $>10$, it is better to perform a surgical hemostasis or TAE with preparation and intensive care for shock during TAE. In cases of TAE failure, re-embolization or surgical hemostasis should be selected according to etiology and vital signs.

There are some limitations in this study. The etiological diseases of obstetric hemorrhage in each case were different and this may affect the success rate of TAE. In addition, most of the patients were transferred to our hospital after delivery and initial treatment in other clinics. Therefore, the general conditions and the hemodynamics on arrival at our hospital were varied. Compounding this problem, because there was no standard protocol for determination, each attending doctors had to make their own decisions per case. Moreover, the numbers of the cases of each group are small. Therefore, future studies should be required more cases to obtain sufficient statistical power.

This TAE protocol is contraindicated for patients with an allergy to iodine contrast medium. Furthermore we couldn't study fully this time, vaginal tears, uterine rupture and bleeding after cesarean delivery may need to be sutured before TAE.

In conclusion, TAE is an effective treatment for obstetric hemorrhage with less invasive, quick completion of hemostasis, and uterine preservation for future pregnancy. TAE is an important intervention and therefore the assessment of each case must be performed accurately but rapidly. For the cases with unstable vital signs before the therapy, careful judgment is necessary. We therefore propose the usage of our new protocol for the determination of intervention with TAE or the surgical hemostasis. We hope that this protocol will rescue many patients by using this at the beginning of the treatments.

\section{Conflict of Interest Statement}

There are no conflicts of interest in connection with this article.

\section{References}

[1] Deneux-Tharaux, C., Berg, C., Bouvier-Colle, M.H., Gissler, M., Harper, M., Nannini, A., Alexander, S., Wildman, K., Breart, G. and Buekens, P. (2005) Under reporting of Pregnancy-Related Mortality in the United States and Europe. Obstetrics \& Gynecology, 106, 684-692. http://dx.doi.org/10.1097/01.AOG.0000174580.24281.e6

[2] Ledee, N., Ville, Y., Musset, D., Mercier, F., Frydman, R. and Fernandez, H. (2001) Management in Intractable Obstetric Haemorrhage: An Audit Study on 61 Cases. European Journal of Obstetrics \& Gynecology and Reproductive Biology, 94, 189-196. http://dx.doi.org/10.1016/S0301-2115(00)00349-3

[3] Ronsmans, C. and Graham, W.J. (2006) Maternal Mortality: Who, When, Where, and Why. Lancet, 368, 1189-1200. http://dx.doi.org/10.1016/S0140-6736(06)69380-X

[4] Brown, B.J., Heaston, D.K., Poulson, A.M., Gabert, H.A., Mineau, D.E. and Miller Jr., F.J. (1979) Uncontrollable Postpartum Bleeding: A New Approach to Hemostasis through Angiographic Arterial Embolization. Obstetrics \& Gynecology, 54, 361-365.

[5] Park, J.K., Shin, T.B., Baek, J.C., Shin, J.K., Choi, W.J., Lee, S.A., Lee, J.H. and Paik, W.Y. (2011) Failure of Uterine Artery Embolization for Controlling Postpartum Hemorrhage. Journal of Obstetrics and Gynaecology Research, 37, 971-978. http://dx.doi.org/10.1111/j.1447-0756.2010.01463.x

[6] Pelage, J.P., Le Dref, O., Jacob, D., Soyer, P., Herbreteau, D. and Rymer, R. (1999) Selective Arterial Embolization of the Uterine Arteries in the Management of Intractable Post-Partum Hemorrhage. Acta Obstetricia et Gynecologica Scandinavica, 78, 698-703. http://dx.doi.org/10.1080/j.1600-0412.1999.780807.X

[7] Pelage, J.P., Le Dref, O., Mateo, J., Soyer, P., Jacob, D., Kardache, M., Dahan, H., Repiquet, D., Payen, D., Truc, J.B., Merland, J.J. and Rymer, R. (1998) Life-Threatening Primary Postpartum Hemorrhage: Treatment with Emergency Selective Arterial Embolization. Radiology, 208, 359-362. http://dx.doi.org/10.1148/radiology.208.2.9680559

[8] Soncini, E., Pelicelli, A., Larini, P., Marcato, C., Monaco, D. and Grignaffini, A. (2007) Uterine Artery Embolization in the Treatment and Prevention of Postpartum Hemorrhage. International Journal of Gynecology \& Obstetrics, 96, 181-185. http://dx.doi.org/10.1016/j.ijgo.2006.12.010

[9] Vegas, G., Illescas, T., Munoz, M. and Perez-Pinar, A. (2006) Selective Pelvic Arterial Embolization in the Management of Obstetric Hemorrhage. European Journal of Obstetrics \& Gynecology and Reproductive Biology, 127, 68-72. http://dx.doi.org/10.1016/j.ejogrb.2005.09.008

[10] Yong, S.P. and Cheung, K.B. (2006) Management of Primary Postpartum Haemorrhage with Arterial Embolisation in 
Hong Kong Public Hospitals. Hong Kong Medical Journal, 12, 437-441.

[11] Tsang, M.L., Wong, W.C., Kun, K.Y., Tai, C.M., Ng, T.K., Lau, K.Y. and Wong, T.P. (2004) Arterial Embolisation in Intractable Primary Post-Partum Haemorrhage: Case Series. Hong Kong Medical Journal, 10, 301-306.

[12] Kobayashi, T., Terao, T., Maki, M. and Ikenoue, T. (2001) Diagnosis and Management of Acute Obstetrical DIC. Seminars in Thrombosis and Hemostasis, 27, 161-167. http://dx.doi.org/10.1055/s-2001-14076

[13] Sentilhes, L., Gromez, A., Clavier, E., Resch, B., Verspyck, E. and Marpeau, L. (2009) Predictors of Failed Pelvic Arterial Embolization for Severe Postpartum Hemorrhage. Obstetrics Gynecology, 113, 992-999. http://dx.doi.org/10.1097/AOG.0b013e3181a114f7

[14] Bros, S., Chabrot, P., Kastler, A., Ouchchane, L., Cassagnes, L., Gallot, D. and Boyer, L. (2012) Recurrent Bleeding within 24 Hours after Uterine Artery Embolization for Severe Postpartum Hemorrhage: Are There Predictive Factors? CardioVascular and Interventional Radiology, 35, 508-514. http://dx.doi.org/10.1007/s00270-011-0181-3

[15] Maassen, M.S., Lambers, M.D., Tutein Nolthenius, R.P., van der Valk, P.H. and Elgersma, O.E. (2009) Complications and Failure of Uterine Artery Embolisation for Intractable Postpartum Haemorrhage. BJOG: An International Journal of Obstetrics and Gynaecology, 116, 55-61. http://dx.doi.org/10.1111/j.1471-0528.2008.01939.x

[16] Cottier, J.P., Fignon, A., Tranquart, F. and Herbreteau, D. (2002) Uterine Necrosis after Arterial Embolization for Postpartum Hemorrhage. Obstetrics Gynecology, 100, 1074-1077.

[17] Hardeman, S., Decroisette, E., Marin, B., Vincelot, A., Aubard, Y., Pouquet, M. and Maubon, A. (2010) Fertility after Embolization of the Uterine Arteries to Treat Obstetrical Hemorrhage: A Review of 53 Cases. Fertility and Sterility, 94, 2574-2579. http://dx.doi.org/10.1016/j.fertnstert.2010.02.052 
Scientific Research Publishing (SCIRP) is one of the largest Open Access journal publishers. It is currently publishing more than 200 open access, online, peer-reviewed journals covering a wide range of academic disciplines. SCIRP serves the worldwide academic communities and contributes to the progress and application of science with its publication.

Other selected journals from SCIRP are listed as below. Submit your manuscript to us via either submit@scirp.org or Online Submission Portal.
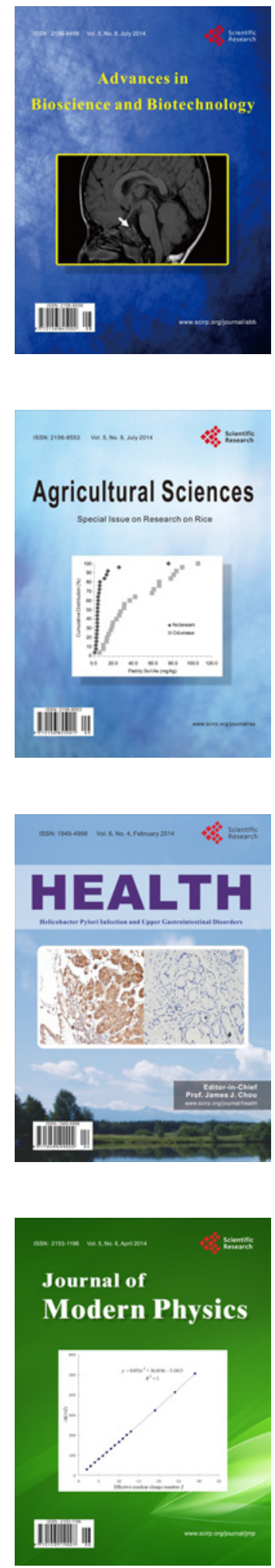
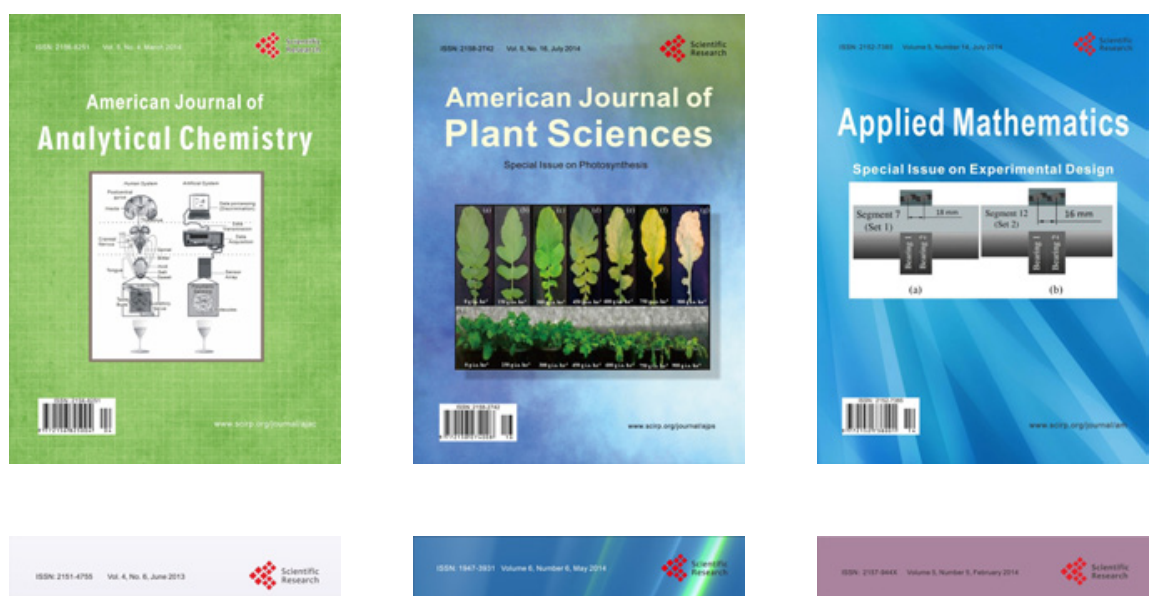

Creative Education
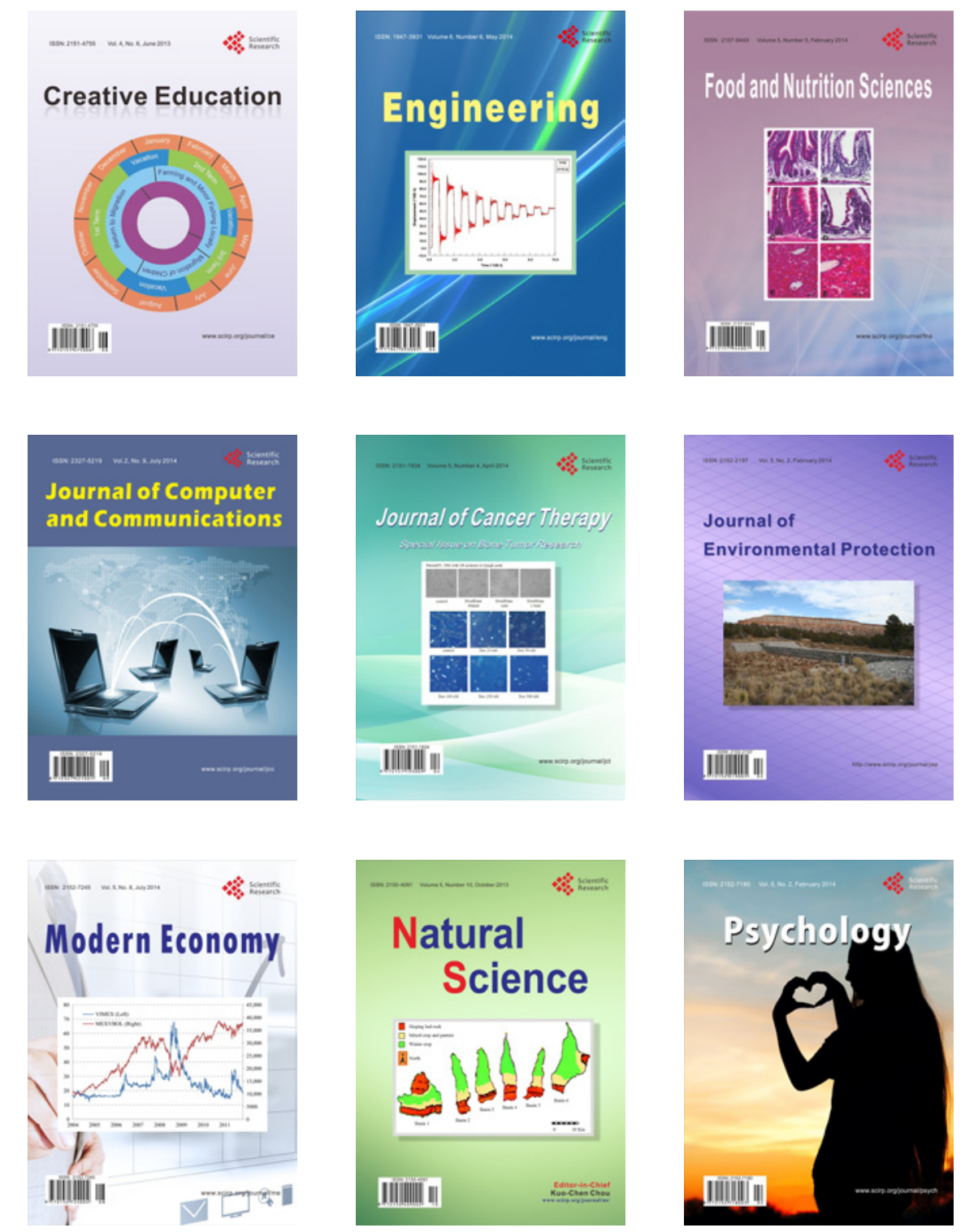